\section{GREGORY W. RUTECKI, MD, Section Editor}

MELDA SONMEZ, MD

Medical Student, Koc University

School of Medicine, Istanbul, Turkey
LOUTFI S. ABOUSSOUAN MD

Department of Pulmonary, Allergy, and Critical Care Medicine, Cleveland Clinic; Associate Professor of Medicine

Cleveland Clinic Lerner College of Medicine of Case Western Reserve University, Cleveland, $\mathrm{OH}$
CAROL FARVER, MD

Department of Pathology, Cleveland Clinic; Professor of Pathology, Cleveland Clinic Lerner College of Medicine of Case Western Reserve University, Cleveland, $\mathrm{OH}$
SUDISH C. MURTHY, MD, PhD

Department of Thoracic and Cardiovascula Surgery, Cleveland Clinic; Professor of Surgery, Cleveland Clinic Lerner College of Medicine of Case Western Reserve University, Cleveland, $\mathrm{OH}$

\section{R00P KAW, MD}

Departments of Hospital Medicine and Outcomes Research

Anesthesiology, Cleveland Clinic; Associate Professor of

Medicine, Cleveland Clinic Lerner College of Medicine of

Case Western University, Cleveland, $\mathrm{OH}$

\title{
Pulmonary infarction due to pulmonary embolism
}

A 76-YEAR-OLD man whose history included $A$ abdominal aortic aneurysm repair, bilateral femoral artery bypass for popliteal artery aneurysm, hypertension, and peptic ulcer disease was admitted to a community hospital with pleuritic chest pain and shortness of breath. Two days earlier, he had undergone repair of a ventral hernia.

At the time of that admission, he reported no fever, chills, night sweats, cough, or history of heart or lung disease. His vital signs were normal, and physical examination had

A 76-year-old man is admitted with pleuritic chest pain and shortness of breath 48 hours after surgery revealed no apparent respiratory distress, no jugular venous distention, normal heart sounds, and no pedal edema; however, decreased air entry was noted in the right lung base. Initial serum levels of troponin and Nterminal pro-B-type natriuretic peptide were normal.

At that time, computed tomographic angiography of the chest showed segmental pulmonary emboli in the left upper and right lower lobes of the lungs and right pleural effusion. Transthoracic echocardiography showed normal atrial and ventricular sizes with no right or left ventricular systolic dysfunction and a left ventricular ejection fraction of $59 \%$.

Treatment with intravenous heparin was started, and the patient was transferred to our hospital.

\section{PLEURAL EFFUSION AND PULMONARY EMBOLISM}

Which of the following is true about pleural effusion?
It is rarely, if ever, associated with pulmonary embolism

Most patients with pleural effusion due to pulmonary embolism do not have pleuritic chest pain Pulmonary embolism should be excluded in all cases of pleural effusion without a clear cause

Pulmonary embolism should be excluded in all cases of pleural effusion that do not have a clear cause. As for the other answer choices:

- Pulmonary embolism is the fourth leading cause of pleural effusion in the United States, after heart failure, pneumonia, and malignancy. ${ }^{1}$

- About $75 \%$ of patients who develop pleural effusion in the setting of pulmonary embolism complain of pleuritic chest pain on the side of the effusion. ${ }^{2}$ Most effusions are unilateral, small, and usually exudative. ${ }^{3}$

\section{EVALUATION BEGINS: RESULTS OF THORACENTESIS}

Our patient continued to receive intravenous heparin.

He underwent thoracentesis on hospital day 3, and 1,000 mL of turbid sanguineous pleural fluid was removed. Analysis of the fluid showed pH 7.27, white blood cell count $3.797 \times 10^{9} / \mathrm{L}$ with $80 \%$ neutrophils, and lactate dehydrogenase (LDH) concentration $736 \mathrm{U} / \mathrm{L}$ (a ratio of pleural fluid LDH to a concurrent serum $\mathrm{LDH}>0.6$ is suggestive of an exudate); the fluid was also sent for culture and cytology. Thoracentesis was terminated early due to cough, and follow- 
up chest radiography showed a moderatesized pneumothorax.

Computed tomography (CT) of the chest at this time showed a small wedge-shaped area of lung consolidation in the right lower lobe (also seen on CT done 1 day before admission to our hospital), with an intrinsic air-fluid level suggesting a focal infarct or lung abscess, now obscured by adjacent consolidation and atelectasis. In the interval since the previous $\mathrm{CT}$, the multiloculated right pleural effusion had increased in size (Figure 1).

\section{THE NEXT STEP}

2 What is the most appropriate next step for this patient?

Consult an interventional radiologist for chest tube placement

Start empiric antibiotic therapy and ask an interventional radiologist to place a chest tube

Start empiric antibiotic therapy, withhold anticoagulation, and consult a thoracic surgeon

Start empiric antibiotic therapy and consult a thoracic surgeon while continuing anticoagulation

The most appropriate next step is to start empiric antibiotic therapy and consult a thoracic surgeon while continuing anticoagulation.

In this patient, it is appropriate to initiate antibiotics empirically on the basis of his significant pleural loculations, a wedge-shaped consolidation, and $80 \%$ neutrophils in the pleural fluid, all of which suggest infection. The unmasking of a wedge-shaped consolidation after thoracentesis, with a previously noted air-fluid level and an interval increase in multiloculated pleural fluid, raises suspicion of a necrotic infection that may have ruptured into the pleural space, a possible lung infarct, or a malignancy. Hence, simply placing a chest tube may not be enough.

Blood in the pleural fluid does not necessitate withholding anticoagulation unless the bleeding is heavy. A pleural fluid hematocrit greater than $50 \%$ of the peripheral blood hematocrit suggests hemothorax and is an indication to withhold anticoagulation. ${ }^{1}$ Our patient's pleural fluid was qualitatively san-

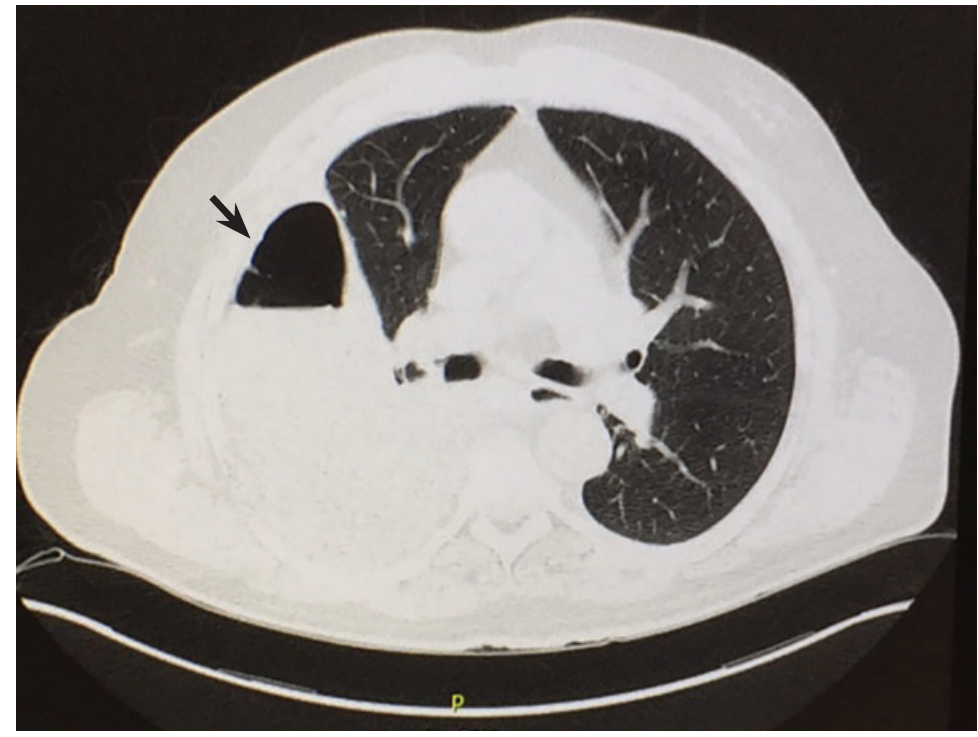

Figure 1. Computed tomography shows a wedge-shaped area of low attenuation suggesting a focal infarction in the collapsed and consolidated right lower lobe.

guineous but not frankly bloody, and therefore we judged that it was not necessary to stop his heparin.

\section{HOW DOES PULMONARY INFARCTION PRESENT CLINICALLY?}

3

Which of the following statements about pulmonary infarction is incorrect?

Cavitation and infarction are more common with larger emboli

Cavitation occurs in fewer than $10 \%$ of pulmonary infarctions

Lung abscess develops in more than 50\% of pulmonary infarctions

Pulmonary thromboembolism is the most common cause of pulmonary infarction

Lung abscess develops in far fewer than 50\% of cases of pulmonary infarction. The rest of the statements are correct.

Cavitation complicates about $4 \%$ to $7 \%$ of infarctions and is more common when the infarction is $4 \mathrm{~cm}$ or greater in diameter. ${ }^{4}$ These cavities are usually single and predominantly on the right side in the apical or posterior segment of the upper lobe or the apical segment of the right lower lobe, as in our patient. ${ }^{5-8} \mathrm{CT}$ demonstrating scalloped inner margins and cross-cavity band
On thoracentesis, $1,000 \mathrm{~mL}$ of turbid sanguineous pleural fluid was removed 


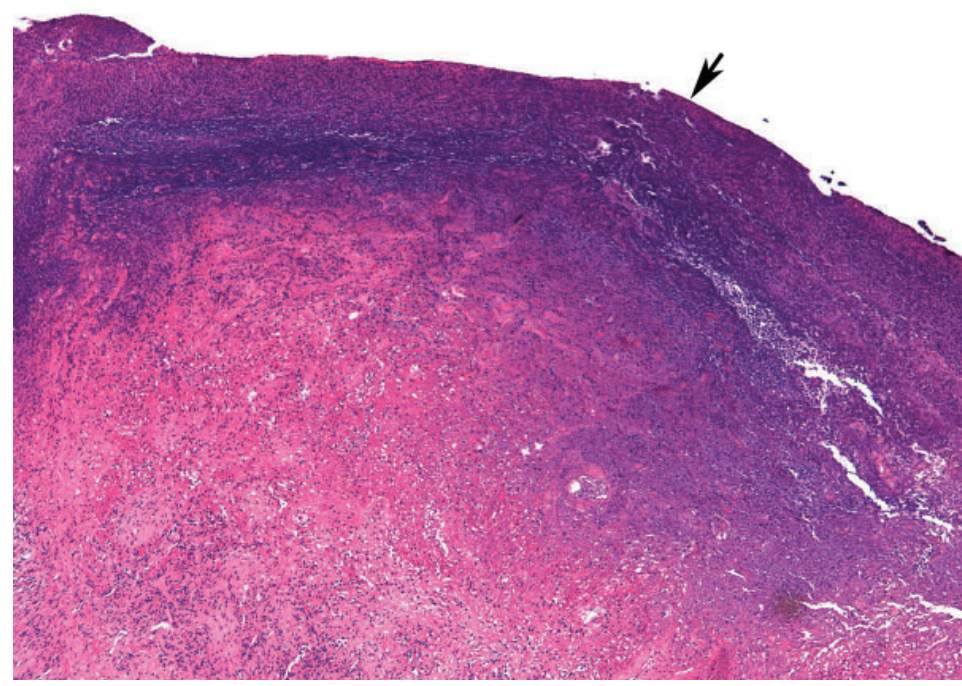

Figure 2. Infarcted lung with alveoli, ischemic necrosis, and a fibrinous exudate on pleural surface (arrow) (hematoxylin and eosin, $\times 12.5$ ).

shadows suggests a cavitary pulmonary infarction..$^{9,10}$

Infection and abscess in pulmonary infarction are poorly understood but have been linked to larger infarctions, coexistent congestion or atelectasis, and dental or oropharyn-
The abscess was resected, the fistula was closed, and chest tubes were placed geal infection. In an early series of 550 cases of pulmonary infarction, 23 patients $(4.2 \%)$ developed lung abscess and $6(1.1 \%)$ developed empyema. ${ }^{11}$ The mean time to cavitation for an infected pulmonary infarction has been reported to be 18 days. ${ }^{12}$

A reversed halo sign, generally described as a focal, rounded area of ground-glass opacity surrounded by a nearly complete ring of consolidation, has been reported to be more frequent with pulmonary infarction than with other diseases, especially when in the lower lobes. ${ }^{13}$

\section{CASE CONTINUED: THORACOSCOPY}

A cardiothoracic surgeon was consulted, intravenous heparin was discontinued, an inferior vena cava filter was placed, and the patient underwent video-assisted thoracoscopy.

Purulent fluid was noted on the lateral aspect of right lower lobe; this appeared to be the ruptured cavitary lesion functioning like an uncontrolled bronchopleural fistula. Two chest tubes, sizes $32 \mathrm{~F}$ and $28 \mathrm{~F}$, were placed af- ter decortication, resection of the lung abscess, and closure of the bronchopleural fistula. No significant air leak was noted after resection of this segment of lung.

Pathologic study showed acute organizing pneumonia with abscess formation; no malignant cells or granulomas were seen (Figure 2). Pleural fluid cultures grew Streptococcus intermedius, while the tissue culture was negative for any growth, including acid-fast bacilli and fungi.

On 3 different occasions, both chest tubes were shortened, backed out $2 \mathrm{~cm}$, and resecured with sutures and pins, and Heimlich valves were applied before the patient was discharged.

Intravenous piperacillin-tazobactam was started on the fifth hospital day. On discharge, the patient was advised to continue this treatment for 3 weeks at home.

The patient was receiving enoxaparin subcutaneously in prophylactic doses; 72 hours after the thorascopic procedure this was increased to therapeutic doses, continuing after discharge. Bridging to warfarin was not advised in view of his chest tubes.

Our patient appeared to have developed a right lower lobe infarction that cavitated and ruptured into the pleural space, causing a bronchopleural fistula with empyema after a recent pulmonary embolism. Other reported causes of pulmonary infarction in pulmonary embolism are malignancy and heavy clot burden, ${ }^{6}$ but these have not been confirmed in subsequent studies. ${ }^{5}$ Malignancy was ruled out by biopsy of the resected portion of the lung, and our patient did not have a history of heart failure. A clear cavity was not noted (because it ruptured into the pleura), but an air-fluid level was described in a wedge-shaped consolidation, suggesting infarction.

\section{How common is pulmonary infarction after pulmonary embolism?}

Pulmonary infarction occurs in few patients with pulmonary embolism. ${ }^{13}$ Since the lungs receive oxygen from the airways and have a dual blood supply from the pulmonary and bronchial arteries, they are not particularly vulnerable to ischemia. However, the reported incidence of pulmonary infarction in pa- 
tients with pulmonary embolism has ranged from $10 \%$ to higher than $30 \%,{ }^{5,14,15}$

The reasons behind pulmonary infarction with complications after pulmonary embolism have varied in different case series in different eras. CT, biopsy, or autopsy studies reveal pulmonary infarction after pulmonary embolism to be more common than suspected by clinical symptoms.

In a Mayo Clinic series of 43 cases of pulmonary infarction diagnosed over a 6 -year period by surgical lung biopsy, 18 (42\%) of the patients had underlying pulmonary thromboembolism, which was the most common cause. $^{16}$

\section{RISK FACTORS} FOR PULMONARY INFARCTION

4 Which statement about risk factors for pulmonary infarction in pulmonary embolism is incorrect?

Heart failure may be a risk factor for pulmonary infarction

Pulmonary hemorrhage is a risk factor for pulmonary infarction

Pulmonary infarction is more common with more proximal sites of pulmonary embolism

$\square$ Collateral circulation may protect against pulmonary infarction

Infarction is more common with emboli that are distal rather than proximal.

Dalen et $\mathrm{al}^{15}$ suggested that after pulmonary embolism, pulmonary hemorrhage is an important contributor to the development of pulmonary infarction independent of the presence or absence of associated cardiac or pulmonary disease, but that the effect depends on the site of obstruction.

This idea was first proposed in 1913, when Karsner and Ghoreyeb ${ }^{17}$ showed that when pulmonary arteries are completely obstructed, the bronchial arteries take over, except when the embolism is present in a small branch of the pulmonary artery. This is because the physiologic anastomosis between the pulmonary artery and the bronchial arteries is located at the precapillary level of the pulmonary artery, and the bronchial circulation does not take over until the pulmonary arterial pressure in the area of the embolism drops to zero.

Using CT data, Kirchner et $\mathrm{al}^{5}$ confirmed that the risk of pulmonary infarction is higher if the obstruction is peripheral, ie, distal.

Using autopsy data, Tsao et $\mathrm{al}^{18}$ reported a higher risk of pulmonary infarction in embolic occlusion of pulmonary vessels less than $3 \mathrm{~mm}$ in diameter.

Collateral circulation has been shown to protect against pulmonary infarction. For example, Miniati et $\mathrm{al}^{14}$ showed that healthy young patients with pulmonary embolism were more prone to develop pulmonary infarction, probably because they had less efficient collateral systems in the peripheral lung fields. In lung transplant recipients, it has been shown that the risk of infarction decreased with development of collateral circulation. ${ }^{19}$

Dalen et $a l,{ }^{15}$ however, attributed delayed resolution of pulmonary hemorrhage (as measured by resolution of infiltrate on chest radiography) to higher underlying pulmonary venous pressure in patients with heart failure and consequent pulmonary infarction. In comparison, healthy patients without cardiac or pulmonary disease have faster resolution of pulmonary hemorrhage when present, and less likelihood of pulmonary infarction (and death in submassive pulmonary embolism).

Data on the management of infected pulmonary infarction are limited. Mortality rates have been as high as $41 \%$ with noninfected and $73 \%$ with infected cavitary infarctions. ${ }^{4}$ Some authors have advocated early surgical resection in view of high rates of failure of medical treatment due to lack of blood supply within the cavity and continued risk of infection.

\section{KEY POINTS}

In patients with a recently diagnosed pulmonary embolism and concurrent symptoms of bacterial pneumonia, a diagnosis of cavitary pulmonary infarction should be considered.

Consolidations that are pleural-based with sharp, rounded margins and with focal areas of central hyperlucencies representing hemorrhage on the mediastinal windows on CT are more likely to represent a pulmonary infarct. ${ }^{20}$

\section{Pulmonary infarction after pulmonary embolism may be more common than suspected by clinical symptoms}




\section{PULMONARY INFARCTION}

\section{REFERENCES}

1. Light RW. Pleural Diseases. 4th ed. Baltimore, MD: Lippincott, Williams \& Wilkins; 2001.

2. Stein PD, Terrin ML, Hales CA, et al. Clinical, laboratory, roentgenographic, and electrocardiographic findings in patients with acute pulmonary embolism and no pre-existing cardiac or pulmonary disease. Chest 1991; 100(3):598-603. pmid:1909617

3. Light RW. Pleural effusion due to pulmonary emboli. Curr Opin Pulm Med 2001; 7(4):198-201. pmid:11470974

4. Libby LS, King TE, LaForce FM, Schwarz MI. Pulmonary cavitation following pulmonary infarction. Medicine (Baltimore) 1985; 64(5):342-348. pmid:4033411

5. Kirchner J, Obermann A, Stuckradt S, et al. Lung infarction following pulmonary embolism: a comparative study on clinical conditions and CT findings to identify predisposing factors. Rofo 2015; 187(6):440-444. doi:10.1055/s-0034-1399006

6. He H, Stein MW, Zalta B, Haramati LB. Pulmonary infarction: spectrum of findings on multidetector helical CT. J Thorac Imaging 2006; 21(1):1-7. doi:10.1097/01.rti.0000187433.06762.fb

7. Scharf J, Nahir AM, Munk J, Lichtig C. Aseptic cavitation in pulmonary infarction. Chest 1971; 59(4):456-458. pmid:5551596

8. Wilson AG, Joseph AE, Butland RJ. The radiology of aseptic cavitation in pulmonary infarction. Clin Radiol 1986; 37(4):327-333. pmid:3731699

9. Butler MD, Biscardi FH, Schain DC, Humphries JE, Blow O, Spotnitz WD. Pulmonary resection for treatment of cavitary pulmonary infarction. Ann Thorac Surg 1997; 63(3):849-850. pmid:9066420

10. Koroscil MT, Hauser TR. Acute pulmonary embolism leading to cavitation and large pulmonary abscess: a rare complication of pulmonary infarction. Respir Med Case Rep 2016; 20:72-74. doi:10.1016/j.rmcr.2016.12.001

11. Levin L, Kernohan JW, Moersch HJ. Pulmonary abscess secondary to bland pulmonary infarction. Dis Chest 1948; 14(2):218-232. pmid:18904835
12. Marchiori E, Menna Barreto M, Pereira Freitas HM, et al. Morpho logical characteristics of the reversed halo sign that may strongly suggest pulmonary infarction. Clin Radiol 2018; 73(5):503.e7-503. e13. doi:10.1016/j.crad.2017.11.022

13. Smith GT, Dexter L, Dammin GJ. Postmortem quantitative studies in pulmonary embolism. In: Sasahara AA, Stein M, eds. Pulmonary Embolic Disease. New York, NY: Grune \& Stratton, Inc; 1965:120-126.

14. Miniati M, Bottai M, Ciccotosto C, Roberto L, Monti S. Predictors of pulmonary infarction. Medicine (Baltimore) 2015; 94(41):e1488. doi:10.1097/MD.0000000000001488

15. Dalen JE, Haffajee Cl, Alpert JS, Howe JP, Ockene IS, Paraskos JA. Pulmonary embolism, pulmonary hemorrhage and pulmonary infarction. N Engl J Med 1977; 296(25):1431-1435. doi:10.1056/NEJM197706232962503

16. Parambil JG, Savci CD, Tazelaar HD, Ryu JH. Causes and presenting features of pulmonary infarctions in 43 cases identified by surgical lung biopsy. Chest 2005; 127(4):1178-1183. doi:10.1378/chest.127.4.1178

17. Karsner HT, Ghoreyeb AA. Studies in infarction: III. The circulation in experimental pulmonary embolism. J Exp Med 1913; 18(5):507-511. pmid:19867725

18. Tsao MS, Schraufnagel D, Wang NS. Pathogenesis of pulmonary infarction. Am J Med 1982; 72(4):599-606. pmid:6462058

19. Burns KE, lacono AT. Incidence of clinically unsuspected pulmonary embolism in mechanically ventilated lung transplant recipients. Transplantation 2003; 76(6):964-968. doi:10.1097/01.TP.0000084523.58610.BA

20. Yousem SA. The surgical pathology of pulmonary infarcts: diagnostic confusion with granulomatous disease, vasculitis, and neoplasia. Mod Pathol 2009; 22(5):679-685. doi:10.1038/modpathol.2009.20

ADDRESS: Roop Kaw MD, Departments of Hospital Medicine and Outcomes Research Anesthesiology, M2 Annex, Cleveland Clinic, 9500 Euclid Avenue, Cleveland, OH 44195; kawr@ccf.org 